\title{
What interventions affect the psychosocial burden experienced by prostate cancer patients undergoing active surveillance? A scoping review
}

\author{
Kim Donachie ${ }^{1} \oplus$ - Erik Cornel ${ }^{2} \cdot$ Thomas Pelgrim $^{3} \cdot$ Leslie Michielsen $^{1} \cdot$ Bart Langenveld $^{4} \cdot$ Marian Adriaansen $^{1}$. \\ Esther Bakker ${ }^{5}$ Lilian Lechner ${ }^{5}$
}

Received: 13 October 2021 / Accepted: 11 January 2022 / Published online: 26 January 2022

(c) The Author(s) 2022

\begin{abstract}
Purpose Living with untreated prostate cancer (PCa) may cause anxiety and uncertainty in men undergoing active surveillance (AS). Developing a psychosocial support program for such patients might promote psychosocial well-being and patient engagement. This review aims to identify interventions with the potential to influence the psychosocial burden of prostate cancer patients undergoing AS.

Methods A scoping review was conducted in accordance with the PRISMA Extension for Scoping Reviews Checklist. A systematic search was conducted in six databases and included publications dating from 2009. All available and eligible evidence was included in this review.

Results After screening 2824 articles, 12 studies were included in the review: nine quantitative, one qualitative, and two mixed method papers. The relative strength of these studies was limited and the quality of most was moderate.

Conclusions The described interventions can be categorized into three major themes: information and education, coping and (psycho)social support, and lifestyle. Psychosocial support for men undergoing AS should entail involvement of family and spouse during the decision-making process, tailored information about PCa treatments, risks, benefits, protocols, lifestyle adjustments, and complementary and alternative medicine. Assessment and promotion of effective coping and selfmanagement strategies are recommended. Healthcare providers should actively promote physical activity and nutritional improvements. Physical activity programs may also be helpful in facilitating peer support, which is especially important for men with limited social support. Future research should investigate combining interventions to increase efficacy and optimize supportive care during AS.
\end{abstract}

Keywords Active surveillance $\cdot$ Diet $\cdot$ Exercise $\cdot$ Lifestyle $\cdot$ Prostate cancer $\cdot$ Psychosocial support

\section{Introduction}

Kim Donachie

kim.donachie@han.nl

1 HAN University of Applied Sciences, Academy of Health, P.O. Box 6960, 6503 GL Nijmegen, The Netherlands

2 Department of Urology, ZGT Twente Hospital, Hengelo, The Netherlands

3 HAN University of Applied Sciences, Nijmegen, The Netherlands

4 Department of Cardiology, Zuyderland Medical Center, Sittard-Geleen, The Netherlands

5 Faculty of Psychology, Open University, Heerlen, The Netherlands
In 2020, approximately 1.4 million men were diagnosed with prostate cancer ( $\mathrm{PCa}$ ) worldwide [1]. The incidence is still increasing and it is estimated that around 2.3 million men will be diagnosed with PCa annually by 2040 [1]. A large proportion of these men are diagnosed with low-risk or insignificant disease [2], and various studies have suggested that men with insignificant or low-risk PCa (LR-PCa) do not benefit from radical treatment. In this patient population, expectant management is warranted [3]. During expectant management, patients do not undergo active treatment but remain under close surveillance. Expectant management can be subdivided into active surveillance (AS) and watchful waiting (WW) [4]. 
AS has a curative intent and consists of aggressive observation to detect early disease progression by monitoring several predefined parameters [4]. If disease progression is detected, radical curative treatment is initiated [5]. In contrast, WW is a palliative option that is most often provided to fragile or older patients when curative or invasive treatment is not desirable (e.g., because of limited life expectancy) [4].

AS is a cost-effective treatment option for LR-PCa [6]. Men who choose AS also avoid complications related to radiotherapy or surgery, such as erectile dysfunction and urinary incontinence [7]. As a result, men undergoing AS generally have greater quality-adjusted life-year scores [8]. Despite these advantages, living with untreated cancer and frequent medical examination may cause anxiety and uncertainty in men undergoing AS [9]. The psychosocial burden of AS has been widely discussed in the literature [10]. A recent study suggests that approximately $30 \%$ of patients are at risk of developing anxiety during the first year of AS [11]. And approximately 5-10\% of men initiate radical treatment based on anxiety without disease progression present $[12,13]$. A decrease in psychosocial well-being may influence patients' adherence to AS and lead them to initiate curative treatment without disease progression $[9,10,14]$.

It has been suggested that there is a greater need for psychosocial support among PCa patients undergoing AS than among patients in active treatment groups [15]. Psychosocial support can be effective in reducing anxiety and uncertainty [16], and providing professional psychosocial support during the first year of AS might enhance its appeal and improve adherence [9-11, 17].

This scoping review aims to identify interventions with the potential to alleviate the psychosocial burden experienced by PCa patients undergoing AS. These interventions include coping strategies, information and psychoeducation, symptom management, lifestyle, complementary and alternative medicine (CAM), self-management, mindfulness, and (peer) support [18]. This research may contribute to developing a psychosocial support program for AS patients that increases psychosocial well-being and patient engagement. This review aimed to answer the following research question: What interventions affect the psychosocial burden experienced by prostate cancer patients undergoing active surveillance?

\section{Methods}

A scoping review was conducted in accordance with the PRISMA Extension for Scoping Reviews Checklist with the aim of being systematic, transparent, and replicable [19]. No ethical approval was required and no protocol was registered.

In contrast to a systematic review, a scoping review provides a more general overview of evidence regarding a broader subject. Scoping reviews are used to inform healthcare practice and map the available evidence on a relevant topic. Since the aim of this review is to identify evidence about interventions that may alleviate psychosocial burden during AS, a scoping review is a suitable design for comprehensively and broadly exploring research on this topic [20, 21].

\section{Search strategy}

Between August and October 2020, a systematic scope search was conducted in the following databases with the assistance of a medical librarian: Cinahl plus with full text, Cochrane, Embase (OVID), Psychinfo (Ebsco), PubMed, and Web of Science. Database-specific search strategies are described in Appendices 1 and 2.

The search strings for all databases are provided in Appendix 1. The results of each search were exported into Rayyan [22], and the deduplication process described by Bramer [23] was performed (Appendices 3, 4, 5, and 6).

The first reviewer (KD) screened all titles and abstracts for mention of prostate cancer, active management (i.e., active surveillance), and interventions influencing psychosocial burden during AS. Articles were either deemed eligible for full-text screening or excluded. A subset of articles was independently screened by blind reviewers (LM, BL, EC).

Following title and abstract screening, full texts of all remaining articles were obtained and read. A selection was then made, based on the inclusion and exclusion criteria. In accordance with the nature of a scoping review, all study types and research designs were included. This included both original studies and reviews that presented new results or interpretations not described in included individual studies.

A hand search of the reference lists of included studies and a forward reference check was conducted to identify additional eligible articles. When hand searching revealed new titles, the process of screening and application of in and exclusion criteria was repeated.

\section{Selection criteria}

In and exclusion criteria were used to assess eligibility for this review. Articles were considered eligible for inclusion if they meet the following criteria:

- A peer-reviewed full-text article was available (reporting empirical qualitative, quantitative, or mixed method studies and literature reviews)

- Articles were published in English or Dutch (for practical reasons)

- Research involved adult male humans (generalizability)

- Publication date was in or after 2009 (when a clear distinction between AS and WW was introduced in clinical practice) $[24,25]$ 
- Research on interventions with a potential effect on psychosocial burden during AS

- Research in men with LR-PCa (AS is justified and safe in LR-PCa)

Articles were excluded from this review for the following criteria:

- Research (primarily/exclusively) on the influence of race, ethnicity, age, socioeconomic status, or health literacy on psychosocial burden (not influenced by a psychosocial support intervention)

- The research population consisted of patients undergoing WW and not AS, or the article made no distinction between WW and AS (WW is a palliative option)

- Research on the influence or effects of focal therapy, i.e., HIFU/cryotherapy (no expectant management)

- Research on the influence of medication on disease progression, i.e., statins or androgen deprivation therapy (no expectant management)

- Research that compared levels of quality of life, distress, anxiety, or uncertainty between treatment groups (does not investigate the effect of interventions on the psychosocial burden within AS treatment group)

- Research on the decision-making process and considerations before beginning AS

\section{Data extraction}

A data extraction sheet was used to derive all relevant data from qualitative and quantitative studies. Hard copy data extraction sheets are stored by the reviewer (KD).

\section{Quality appraisal}

Quality appraisal tools were used to assess the quality of the included evidence and assess the level of evidence [26-29]. The quality of included studies was ranked as "high," "moderate," or "low." Two reviewers (KD, BL) discussed various cutoff values until consensus was reached. This corresponds with the scores described in Online Resource 1.

\section{Results}

\section{Search results}

The literature search was carried out in six scientific databases and yielded 2808 unique citations. A supplementary hand search identified 16 more citations. A flowchart of the search strategy is provided in Fig. 1.

Blind reviewers (LM, BL, EC) independently screened a random subset of 1100 articles. An $82.5 \%$ consensus rate on this subset was established. The remaining conflicting results were discussed by the reviewers until consensus was reached.

\section{Characteristics of the studies}

After the full-text screening, 12 studies met all criteria and were included in the review [30-41]. This included nine quantitative [30-33, 35, 39-41], one qualitative [37], and two mixed method $[34,36]$ papers. All studies involved men with LR-PCa who were under active management. An overview of study characteristics is provided in Table 1.

\section{Quality appraisal}

The results of the quality appraisal are provided in Table 2 . None of the 12 studies met all quality criteria, and only three met more than $80 \%$ of the quality criteria and were considered to be of high quality $[30,34,40]$.

\section{Themes}

Three major intervention themes emerged from this review: information and education, coping and (psycho)social support, and lifestyle. A narrative synthesis of the results from the included studies is provided.

\section{Information and education}

Five studies investigated the relationship between information, education, and the psychosocial burden of AS [32-36]. These studies confirmed the presence of unmet informational needs among PCa patients eligible for, or currently undergoing, AS.

The systematic review carried out by McIntosh et al. identified unmet informational needs [36]. PCa patients undergoing AS often receive information that is inadequate, confusing, and inconsistent, which causes them distress. They receive inadequate information about $\mathrm{PCa}$, signs of progression, future treatment options, and adjuvant treatment such as diet and exercise.

Ideally, the information should be consistent, unambiguous, concise, and adequately tailored to the individual. This conclusion was supported by the systematic review conducted by Kinsella et al., which identified facilitators and barriers to AS adherence [34]. Their review affirmed the importance of customization and personalization of just-intime information. It also stressed that information should not only be provided to patients, but education must involve partners and family. Inaccurate risk assessment can cause family and friends to pressure patients to undergo curative treatment. 
Fig. 1 PRISMA flowchart

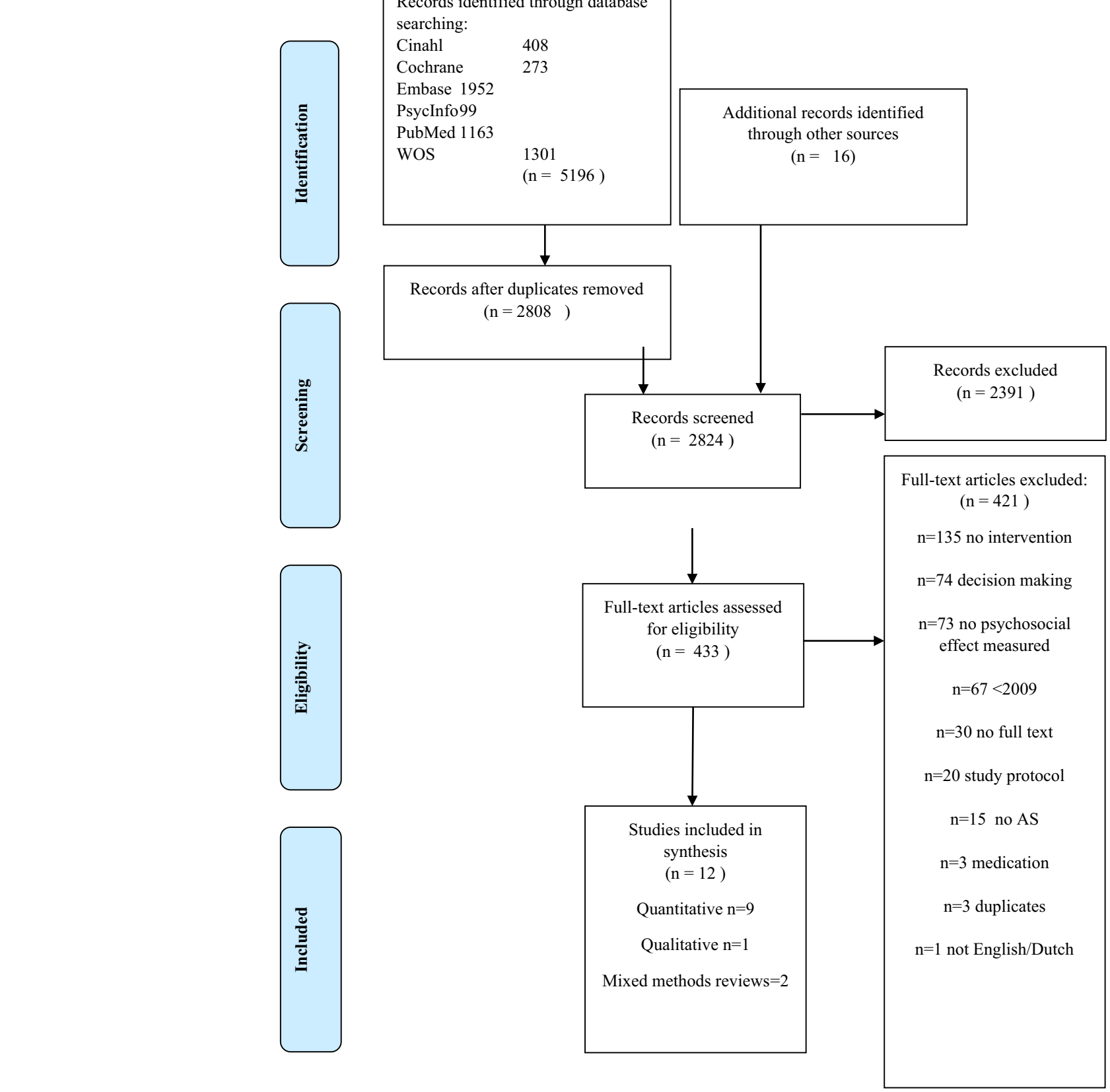

The importance of educating patients and their partners was affirmed by the results of the quasi-experiment conducted by Hedden et al. [32], which evaluated the effect of an educational intervention on distress and anxiety among men diagnosed with LR-PCa $(n=71)$ and their partners $(n=48)$. The study demonstrated that partners had higher distress levels than patients before attending an educational seminar $(p=0.03)$ about PCa and treatments. That seminar addressed the informational needs identified by McIntosh et al. [36]. Customized and personalized information was also provided during a private meeting between the patient, partner, urologist, and radio-oncologist. Hedden et al. found that attending the seminar and private meeting significantly decreased distress among patients and partners compared to their distress before the seminar $(p=<0.001)$.

Kinsella et al. assessed the long-term effects of a seminar on AS adherence [35]. They conducted a quasi-experiment to assess the effect of a single 1.5-h educational seminar, after which time was scheduled for questions and peer discussion. During the first year of the study, no one experienced clinical disease progression in the intervention $(n=120)$ or control ( $n=135)$ group. However, a significantly 


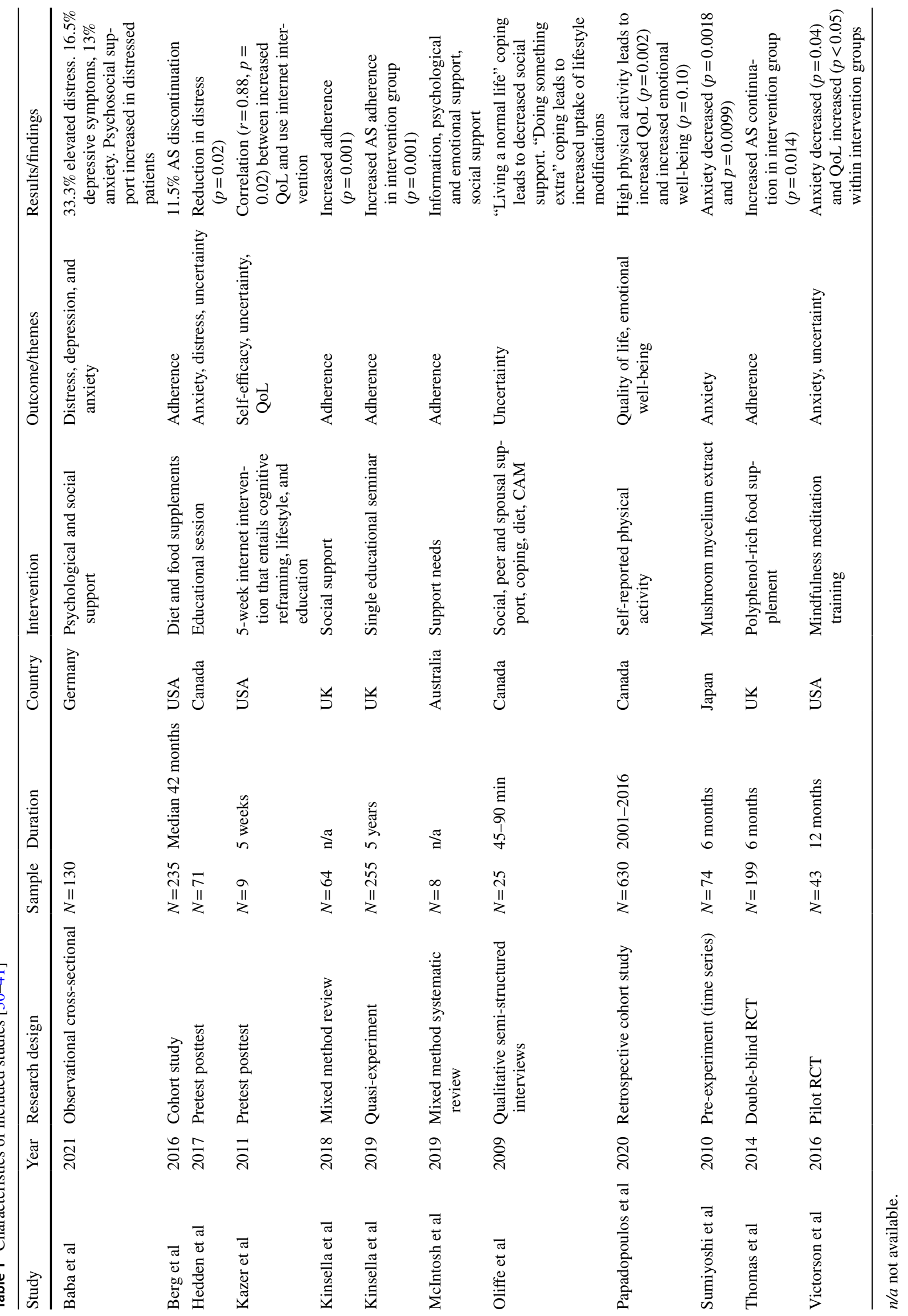


Table 2 Quality scores and levels of evidence

\begin{tabular}{lllllll}
\hline Study & Year & Research design & Quality tool & Quality score & Quality appraisal & Level of evidence \\
\hline Baba et al & 2021 & Observational cross-sectional & Law et al & 11 & High & Level VI \\
Berg et al & 2016 & Cohort study & Law et al & 9 & Moderate & Level IV \\
Hedden et al & 2017 & Pretest posttest & Law et al & 11 & Moderate & Level III \\
Kazer et al & 2011 & Pretest posttest & Law et al & 11 & Moderate & Level III \\
Kinsella et al & 2019 & Quasi-experiment & Law et al & 9 & Moderate & Level III \\
Kinsella et al & 2018 & Mixed method review & Hong et al & 14 & High & Level V \\
McIntosh et al & 2019 & Mixed method systematic review & Hong et al & 13 & Moderate & Level V \\
Oliffe et al & 2009 & Qualitative semi-structured interviews & Tong et al & 20 & Moderate & Level VI \\
Papadopoulos et al & 2020 & Retrospective cohort study & Law et al & 11 & Moderate & Level IV \\
Sumiyoshi et al & 2010 & Pre-experiment (time series) & Law et al & 10 & Moderate & Level III \\
Thomas et al & 2014 & Double-blind RCT & Law et al & 13 & High & Level II \\
Victorson et al & 2016 & Pilot RCT & Law et al & 10 & Moderate & Level II \\
\hline
\end{tabular}

smaller proportion of men in the intervention group (11\%, $p=0.003$ ) dropped out of AS compared to the control group $(25 \%)$. After 5 years, the intervention group continued to demonstrate increased adherence to AS with a significantly smaller dropout rate among patients with no evidence of disease progression $(p<0.001)$.

Kazer et al. conducted a quasi-experimental study to investigate the effect of an internet-based information and education intervention on older men undergoing AS (aged $66-79, x=72$ ) [33]. The intervention was combined with tailored information provided via email by a specialized nurse upon request. Cognitive reframing and self-management strategies were also provided. After subjects completed the intervention, researchers observed an overall trend toward an increase in quality of life (QoL). This study suggests that it is feasible to provide information online.

Successful reassurance and education of the patient and family are key facilitators to AS adherence. Health literacy also plays an important role, and provision and access to relevant and understandable information is a consistent theme. Inaccurate risk and survival perceptions by patients and their families may increase distress and decrease AS uptake and adherence. In contrast, distress is decreased and AS uptake and adherence are increased by the provision of transparent, consistent, and understandable information regarding PCa diagnosis, prognosis, treatment options, active surveillance, monitoring protocols, AS-associated risks, and reliable guidelines on complementary options, diet, and lifestyle changes.

\section{Coping and (psycho)social support}

Five studies addressed the importance of coping and social support during AS [30, 33, 34, 36, 37]. A cross-sectional study conducted by Baba et al. found that distressed patients undergoing AS have an elevated need for psychosocial support [30]. The systematic review conducted by McIntosh et al. found that distress is caused by unmet emotional, psychological, and social support needs [36]. In particular, men who experience AS negatively have more difficulty dealing with AS. That was less complicated for men who felt that AS was "having a positive impact," according to Kinsella et al. [34]. They found that such men experience less uncertainty and feel more in control. Baba et al. found something similar in their cross-sectional study of men with PCa $(n=130)$ who were undergoing AS $(n=19)$ [30]. They claimed that a "positive attitude" was helpful to promote coping with ASassociated distress.

Oliffe et al. affirmed the importance of adopting coping strategies [37]. Their qualitative interview study identified two self-management strategies for alleviating AS-induced uncertainty in a sample of 25 men: "living a normal life" and "doing something extra." The study also assessed the implications of these strategies. Although "living a normal life" seemed to be an effective strategy for avoiding worry and distress, it was associated with a limited support network, avoidance, stoicism, and a resistance to lifestyle changes. Thus, this strategy may decrease AS compliance. In contrast, adopting a "doing something extra" strategy seemed to promote positive lifestyle changes. This strategy is characterized by engagement in self-health and education. Oliffe et al. observed an increased use of CAM in this population. Engagement with their partner, family, and friends seemed to affirm the patients' commitment to AS and increase adherence.

The finding that social support promotes coping with AS corresponds with the results from other studies in this review. The review conducted by Kinsella et al. demonstrated a strong correlation between AS adherence and AS support groups for men and their families [34]. This was confirmed by Baba et al., whose study indicated that peer support from interpersonal relationships with partners, 
friends, and family helps patients cope with AS [30]. McIntosh et al. recommend referring men to (online anonymous) support groups during AS [36].

Psychotherapeutic interventions may help men who are unable to cope with AS. Kazer et al. investigated a cognitive reframing behavioral intervention among men $(n=9)$ and found a positive correlation between intervention usage and QoL, especially in the domains "role function related to emotional health" and "social function" ( $r=0.88, p=0.02)$ [33].

In summary, men who have unmet support needs during AS are at increased risk of distress. Adopting helpful coping and self-management strategies, such as a positive attitude and a "doing something extra" strategy, may improve patients' ability to cope with AS. Furthermore, social support promotes coping, AS adherence, and commitment. Social support can be present within personal relationships or facilitated through peer support groups. Professional psychotherapeutic help may be beneficial to men who feel unable to cope with AS.

\section{Lifestyle}

Eight studies examined the uptake and effects of lifestyle adjustments [30, 31, 33, 34, 37-41]. Most of them focused on diet and food supplements [31, 33, 34, 39, 40]. According to Oliffe et al., engagement in self-health is associated with increased commitment and adherence to AS [37]. The review conducted by Kinsella et al. found that self-management strategies, such as exercise and stress management, help men cope with uncertainty during AS [34]. Baba et al. also stated that physical activity was helpful for men who were dealing with distress [30].

These findings were confirmed by the retrospective cohort study conducted by Papadopoulos et al. [38], which explored the association between self-reported physical activity, QoL, and emotional well-being in a cohort of 630 men. Highly active participants showed significantly higher $(p=0.002)$ QoL scores. The most active group also demonstrated the highest odds of experiencing high emotional well-being $(p=0.010)$.

Kazer et al. investigated the effect of an internet-based intervention that combined various components, including nutritional recommendations and the promotion of physical activity [33]. This incorporated weight control, exercise, limiting meat, and avoiding alcohol and smoking. Their fourcomponent intervention resulted in an overall trend toward improved QoL. After patients completed the intervention period, their QoL returned toward baseline, indicating that the intervention had a temporary and transient effect.

Diet and nutritional recommendations were also investigated in the large longitudinal cohort study conducted by Berg et al. [31]. They examined the effect of a holistic AS approach on adherence in a sample of 235 men. Holistic AS consisted of AS monitoring complemented with strict dietary recommendations. In this study, with a median follow-up of 42 months, only $1.3 \%$ of patients discontinued AS because of anxiety or uncertainty. In general, researchers observe a dropout of 5-10\% due to anxiety and uncertainty [13].

Thomas et al. conducted a double-blind placebo-controlled randomized trial to evaluate the effect of a polyphenol-rich whole food supplement on AS discontinuation [40]. They found that a statistically significantly lower proportion of men in the food supplement group $(n=134)$ discontinued AS $(p=0.014)$. Their reasons for discontinuing AS were not disclosed.

Only the study by Sumiyoshi et al. assessed the direct effect of a food supplement on psychosocial well-being [39]. They examined the effect of dietary administration of mushroom mycelium extract (AHCC) on anxiety during a quasiexperiment among 74 men treated with expectant management. Significantly lower State-Trait Anxiety Index (STAI) scores were observed in participants with high anxiety levels before the intervention $(p<0.01)$.

Anxiety and uncertainty also significantly decreased as a result of participation in an 8-week mindfulness meditation training, according to Victorson et al. [41]. Results from their pilot randomized controlled trial demonstrated a significant $(p=0.04)$ within-group decrease of anxiety in the intervention group $(n=24)$ between baseline and 6 months after the intervention. Uncertainty decreased between baseline and 12 months $(p<0.01)$, and global mental health increased between baseline and 8 weeks $(p<0.05)$. A between-group analysis indicated that post-traumatic growth was the outcome that significantly increased in the intervention group ( $p=0.01)$ compared to the control group $(n=19)$.

Overall, engaging in self-health by making healthy lifestyle adjustments seems to provide men with important selfmanagement strategies during AS, resulting in decreased distress, anxiety, and uncertainty and improved AS adherence. In addition, some lifestyle adjustments seem to directly affect anxiety and uncertainty. For instance, exercise seems to directly benefit emotional well-being, and participation in a mindfulness meditation program decreased anxiety and uncertainty.

\section{Discussion}

This scoping review identified interventions that affect the psychosocial burden experienced by PCa patients undergoing AS. Those interventions can be categorized into three major themes: information and education, coping and psychosocial support, and lifestyle. 
In the first category, providing information about $\mathrm{PCa}$, treatments, AS protocols, prognosis, mortality, morbidity, and lifestyle via a website or educational seminar seems to assist in risk assessment and decrease uncertainty [32, 36]. A positive impact on AS adherence is associated with these interventions. The involvement of the patient's partner and family is critical to the provision of information since they seem to play an important role in reassuring the patient and facilitating AS adherence [32, 36]. In addition, the information should be tailored to the needs and health literacy levels of the patient and their family [34]. Individual meetings or tailored contacts could provide opportunities to assess whether patients have obtained all the required information and whether that information was consistent, understandable, and relevant [33].

Selecting AS should be the result of a thorough consideration of risks, benefits, and coping and self-management strategies. It should not merely be selected to avoid treatment-related complications. Thus, it is recommended that shared decision-making techniques and decision aids be used to support careful deliberation about AS [42-44].

The second category comprised coping and psychosocial support interventions [30, 33-35, 37]. Adopting a positive attitude toward AS seems to decrease distress and uncertainty [30,34]. Although an avoidant self-management strategy enables patients to temporarily prevent stress, uncertainty, and anxiety associated with AS, this strategy is also associated with a limited support network and a resistance to lifestyle changes [33,37]. These last two features are negatively associated with AS adherence. Adopting a self-management strategy that emphasizes self-health, selfeducation, and engagement with family and friends seems to improve AS adherence.

It is recommended to assess the adoption of effective coping and self-management strategies. Healthcare providers can use specially designed questionnaires to identify anxiety, uncertainty, or distress [45-47], and professional psychological support should be offered to men who are unable to cope with AS [36]. Cognitive behavior therapy, especially cognitive reframing, might help to promote a positive attitude [33]. Social support from peers, family, and friends also plays an important role in helping patients deal with the psychosocial burden associated with AS. Some patients may benefit from peer support, especially those who have a limited social support network or who do not wish to discuss their experience with their friends or family [30, 34, 36, 37].

Lifestyle interventions and adjustments comprise the final category [30, 31, 33, 34, 37-41]. Physical activity, exercise, and mindfulness seem to directly affect distress, QoL, and uncertainty [30, 33, 34, 38, 41]. In addition, dietary recommendations and nutritional supplements may positively influence AS adherence [31, 33, 34, 39, 40]. It is most likely that lifestyle adjustments provide a self-management strategy that helps patients cope with uncertainty during AS [37]. Engaging in self-health practices seems to promote a sense of control over one's disease that directly and indirectly affects the psychosocial burden experienced during AS. Patients and their partners actively seek information about dietary and exercise interventions [48]. Providing such recommendations may lead some men to make positive lifestyle changes. However, others may need professional support and guidance (e.g., from a nutritional or physical therapist) to facilitate lifestyle improvements. Motivational interviewing might improve the uptake of healthy lifestyle changes $[49,50]$.

\section{Study strengths and limitations}

The results of this review are based on a comprehensive and systematic search of multiple databases. The search and selection processes have been described in detail. In addition, the quality of the available evidence was assessed and a quality appraisal is provided. Despite the strengths of this scoping review, it has some limitations. One is that it was conducted by a single researcher. However, three independent reviewers were involved in selecting the articles to be reviewed. The methodology and process also were extensively discussed within the supervisory team. This decreases the risk of measurement bias.

A second limitation is the quality of the included evidence. None of the included studies met all quality criteria. In addition, the level of evidence of the included studies was predominantly low to medium. Of the two high-level studies included, one was a pilot study that therefore had a small sample size. This may have influenced the validity of the results. However, this review imposed no restrictions on research designs and thus included all available and eligible evidence in this review.

In addition, this review identified interventions that mostly had a short-term, positive effect [33], and most of the studies investigated stand-alone interventions. Therefore, it is unclear whether combining interventions can have a complementary effect and whether a combination of interventions would generate longer-lasting or improved results (e.g., a combination of interventions that have a transitory effect with interventions that demonstrate long-term results) [35].

\section{Conclusion}

In conclusion, various psychosocial interventions and lifestyle adjustments seem to affect the psychosocial burden experienced by prostate cancer patients undergoing active surveillance. Based on the findings from this review, various interventions appear to decrease the psychosocial burden during AS. Firstly, this review emphasizes 
the importance of transparent, understandable, relevant, and reliable information about $\mathrm{PCa}$, treatments, AS risks, benefits, and protocols. In addition, information on lifestyle adjustments and CAM should be provided. Secondly, families and partners should receive adequate information and be involved in the decision-making process. Moreover, assessing the coping and self-management strategies used by men undergoing AS can identify those who find it difficult to handle. Men who are struggling with AS or have limited coping skills should be encouraged to adopt helpful coping strategies, such as a positive attitude and health-promoting behavior. Professional psychological support should be offered to men unable to deal with AS. Furthermore, healthcare providers (e.g., urologists, nurse specialists, nurses) should actively promote physical activity and nutritional improvements by providing exercise and dietary recommendations or organizing lifestyle support programs. Physical activity programs for men undergoing AS may also be helpful in facilitating peer support.

In regard to the limitations of this review, the included number of studies, and their quality, it is recommended that future research investigates the feasibility and efficacy of combining various interventions that may potentially decrease psychosocial burden and optimize supportive care during AS.

Supplementary Information The online version contains supplementary material available at https://doi.org/10.1007/s00520-022-06830-z.

Author contribution All authors contributed to the study concept and design. Conceptualization and study design selection were conducted by KD, MA, EC, EB, and LL. Data collection was performed by KD and TP. Screening and filtering were conducted by KD, BL, EC, and LM. The first draft of the manuscript was written by KD and all authors commented on previous versions of it. All authors read and approved the final manuscript.

Funding The authors disclose receipt of financial support for the research from the Dutch Research Council [grant number 023.016.048].

Data availability The data that support the findings of this study are available on request from the corresponding author, KD.

Code availability Not applicable.

\section{Declarations}

Ethics approval Since this is a review study, no ethical approval is required.

Consent to participate Not applicable.

Consent for publication Not applicable.

Conflict of interest The authors declare no competing interests.
Open Access This article is licensed under a Creative Commons Attribution 4.0 International License, which permits use, sharing, adaptation, distribution and reproduction in any medium or format, as long as you give appropriate credit to the original author(s) and the source, provide a link to the Creative Commons licence, and indicate if changes were made. The images or other third party material in this article are included in the article's Creative Commons licence, unless indicated otherwise in a credit line to the material. If material is not included in the article's Creative Commons licence and your intended use is not permitted by statutory regulation or exceeds the permitted use, you will need to obtain permission directly from the copyright holder. To view a copy of this licence, visit http://creativecommons.org/licenses/by/4.0/.

\section{References}

1. Sung H, Ferlay J, Siegel RL, Laversanne M, Soerjomataram I, Jemal A et al (2021) Global cancer statistics 2020: GLOBOCAN estimates of incidence and mortality worldwide for 36 cancers in 185 countries. CA Cancer J Clin 71(3):209-249. https://doi.org/10.3322/caac.21660

2. Cooperberg M, Lubeck D, Meng M, Mehta S, Carroll P (2004) The changing face of low-risk prostate cancer: trends in clinical presentation and primary management. J Clin Oncol 22(11):2141-2149

3. Serrell E, Pitts D, Hayn M, Beaule L, Hansen M, Sammon J (2018) Review of the comparative effectiveness of radical prostatectomy, radiation therapy, or expectant management of localized prostate cancer in registry data. Urol Oncol 36(4):183-192

4. Filson C, Marks L, Litwin M (2015) Expectant management for men with early stage prostate cancer. CA Cancer J Clin 65(4):264-282

5. Mottet N, van den Bergh RC, Briers E, Van den Broeck T, Cumberbatch MG, De Santis M. et al (2021) EAU-EANM-ESTROESUR-SIOG guidelines on prostate cancer-2020 update. Part 1: screening, diagnosis, and local treatment with curative intent. Euro Urol 79(2):243-262

6. Keegan K, Dall'Era M, Durbin-Johnson B, Evans C (2011) Active surveillance for prostate cancer compared with immediate treatment. Cancer 118(14):3512-3518

7. Hamdy F, Donovan J, Lane J, Mason M, Metcalfe C, Holding P et al (2020) Activemonitoring, radical prostatectomy and radical radiotherapy in PSA-detected clinically localised prostate cancer: the ProtecT three-arm RCT. Health Technology Assessment (Winchester, England), 24(37): 1

8. Hayes J, Ollendorf D, Pearson S, Barry M, Kantoff P, Stewart S et al (2010) Active surveillance compared with initial treatment for men with low-risk prostate cancer. JAMA 304(21):2373

9. Pickles T, Ruether J, Weir L, Carlson L, Jakulj F (2007) Psychosocial barriers to active surveillance for the management of early prostate cancer and a strategy for increased acceptance. BJU Int 100(3):544-551

10. Kinsella N, Stattin P, Cahill D, Brown C, Bill-Axelson A, Bratt $O$ et al (2018) Factors influencing men's choice of and adherence to active surveillance for low-risk prostate cancer: a mixedmethod systematic review. Eur Urol 74(3):261-280

11. Marzouk K, Assel M, Ehdaie B, Vickers A (2018) Long-term cancer specific anxiety in men undergoing active surveillance of prostate cancer: findings from a large prospective cohort. J Urol 200(6):1250-1255

12. Venderbos LD, Van Den Bergh RC, Roobol MJ, Schröder FH, Essink-Bot ML, Bangma CH, ..., Korfage IJ (2015) A longitudinal study on the impact of active surveillance for prostate cancer on anxiety and distress levels. Psychooncology 24(3):348-354 
13. Albertsen P (2015) Observational studies and the natural history of screen-detected prostate cancer. Curr Opin Urol 25(3):232-237

14. Parker P, Davis J, Latini D, Baum G, Wang X, Ward J et al (2015) Relationship between illness uncertainty, anxiety, fear of progression and quality of life in men with favourable-risk prostate cancer undergoing active surveillance. BJU Int 117(3):469-477

15. Cheah WL, Ling NC, Chang KH (2016) The supportive care needs for prostate cancer patients in Sarawak. Chinese Clinical Oncol 5(1):7-7

16. Osborn R, Demoncada A, Feuerstein M (2006) Psychosocial interventions for depression, anxiety, and quality of life in cancer survivors: meta-analyses. Int J Psychiatry Med 36(1):13-34

17. Parahoo K, McDonough S, McCaughan E, Noyes J, Semple C, Halstead E et al (2015) Psychosocial interventions for men with prostate cancer: a Cochrane systematic review. BJU Int 116(2):174-183

18. Donachie K, Cornel E, Adriaansen M, Mennes R, Oort I, Bakker E et al (2020) Optimizing psychosocial support in prostate cancer patients during active surveillance. Int J Urol Nurs 14(3):115-123

19. Tricco A, Lillie E, Zarin W, O'Brien K, Colquhoun H, Levac D et al (2018) PRISMA Extension for Scoping Reviews (PRISMA-ScR): checklist and explanation. Ann Intern Med 169(7):467-473

20. Munn Z, Peters M, Stern C, Tufanaru C, McArthur A, Aromataris E (2018) Systematic review or scoping review? Guidance for authors when choosing between a systematic or scoping review approach. BMC Med Res Methodol 18(1):1-7

21. Peterson J, Pearce P, Ferguson L, Langford C (2017) Understanding scoping reviews. J Am Assoc Nurse Pract 29(1):12-16

22. Ouzzani M, Hammady H, Fedorowicz Z, Elmagarmid A (2016) Rayyan - a web and mobile app for systematic reviews. Syst Rev 5(1):1-10

23. Bramer W, Giustini D, De Jonge G, Holland L, Bekhuis T (2016) De-duplication of database search results for systematic reviews in EndNote. J Med Libr Assoc 104(3): 240

24. Wallace M, Bailey D, O'Rourke M, Galbraith M (2004) The watchful waiting management option for older men with prostate cancer: state of the science. Oncol Nurs Forum 31(6):1057-1066

25. Adolfsson J (2008) Watchful waiting and active surveillance: the current position. BJU Int 102(1):10-14

26. Law M, Stewart D, Letts L, Pollock N, Bosch J, Westmorland M (1998) Guidelines for critical review of qualitative studies. McMaster University occupational therapy evidence-based practice research Group, 1-9

27. Hong QN, Fàbregues S, Bartlett G, Boardman F, Cargo M, Dagenais P. et al (2018) The mixed methods appraisal tool (MMAT) version 2018 for information professionals and researchers. Educ Info 34(4):285-291

28. Tong A, Sainsbury P, Craig J (2007) Consolidated criteria for reporting qualitative research (COREQ): a 32-item checklist for interviews and focus groups. Int J Qual Health Care 19(6):349-357

29. Melnyk BM, Fineout-Overholt E (eds) (2011) Evidence-based practice in nursing \& healthcare: a guide to best practice. Lippincott Williams \& Wilkins

30. Baba N, Schrage T, Hartmann A, Baba K, Wuensch A, SchultzeSeemann W, Joos A (2021) Mental distress and need for psychosocial support in prostate cancer patients: an observational crosssectional study. Int J Psychiatry Med 56(1):51-63

31. Berg CJ, Habibian DJ, Katz AE, Kosinski KE, Corcoran AT, Fontes AS (2016) Active holistic surveillance: the nutritional aspect of delayed intervention in prostate cancer. J Nutr Metab

32. Hedden L, Wassersug R, Mahovlich S, Pollock P, Sundar M, Bell RH, ..., Higano CS (2017) Evaluating an educational intervention to alleviate distress amongst men with newly diagnosed prostate cancer and their partners. BJU Int 120(5B):E21-E29
33. Kazer MW, Bailey DE, Sanda M, Colberg J, Kelly WK (2011) An internet intervention for management of uncertainty during active surveillance for prostate cancer. In Oncol Nurs Forum 38(5):561-568

34. Kinsella N, Stattin P, Cahill D, Brown C, Bill-Axelson A, Bratt O, ..., Van Hemelrijck M (2018) Factors influencing men's choice of and adherence to active surveillance for low-risk prostate cancer: a mixed-method systematic review. Eur Urol 74(3):261-280

35. Kinsella N, Beckmann K, Cahill D, Elhage O, Popert R, Cathcart P, ..., Van Hemelrijck M (2019) A single educational seminar increases confidence and decreases dropout from active surveillance by 5 years after diagnosis of prostate cancer. Eur Urol Oncol 2(4):464-470

36. McIntosh M, Opozda MJ, Evans H, Finlay A, Galvão DA, Chambers SK, Short CE (2019) A systematic review of the unmet supportive care needs of men on active surveillance for prostate cancer. Psychooncology 28(12):2307-2322

37. Oliffe JL, Davison BJ, Pickles T, Mróz L (2009) The self-management of uncertainty among men undertaking active surveillance for low-risk prostate cancer. Qual Health Res 19(4):432-443

38. Papadopoulos E, Alibhai SM, Doré I, Matthew AG, Tomlinson GA, Nesbitt M, ..., Santa Mina D (2020) Associations between selfreported physical activity, quality of life, and emotional well-being in men with prostate cancer on active surveillance. Psychooncology 29(6):1044-1050

39. Sumiyoshi Y, Hashine K, Kakehi Y, Yoshimura K, Satou T, Kuruma $\mathrm{H}$, ..., Shinohara N (2010) Dietary administration of mushroom Mycelium extracts in patients with early stage prostate cancers managed expectantly: a phase II study. Jpn J Clin Oncol 40(10):967-972

40. Thomas R, Williams M, Sharma H, Chaudry A, Bellamy P (2014) A double-blind, placebo-controlled randomised trial evaluating the effect of a polyphenol-rich whole food supplement on PSA progression in men with prostate cancer-the UK NCRN Pomi-T study. Prostate Cancer Prostatic Dis 17(2):180-186

41. Victorson D, Hankin V, Burns J, Weiland R, Maletich C, Sufrin N, ..., Brendler C (2017) Feasibility, acceptability and preliminary psychological benefits of mindfulness meditation training in a sample of men diagnosed with prostate cancer on active surveillance: results from a randomized controlled pilot trial. Psycho-Oncology 26(8):1155-1163

42. Bayliss D (2016) Decision-making in prostate cancer: active surveillance over other Treatment Options. Urol Nurs 36(3)

43. Loeb S, Folkvaljon Y, Curnyn C, Robinson D, Bratt O, Stattin P (2017) Uptake of active surveillance for very-low-risk prostate cancer in Sweden. JAMA Oncol 3(10):1393-1398

44. Collée GE, van der Wilk BJ, van Lanschot JJB, Busschbach JJ, Timmermans L, Lagarde SM, Kranenburg LW (2020) Interventions that facilitate shared decision-making in cancers with active surveillance as treatment option: a systematic review of literature. Curr Oncol Rep 22(10):1-13

45. Bailey DE Jr, Wallace M, Latini DM, Hegarty J, Carroll PR, Klein EA, Albertsen PC (2011) Measuring illness uncertainty in men undergoing active surveillance for prostate cancer. Appl Nurs Res 24(4):193-199

46. Chambers SK, Zajdlewicz L, Youlden DR, Holland JC, Dunn J (2014) The validity of the distress thermometer in prostate cancer populations. Psychooncology 23(2):195-203

47. van den Bergh RC, Korfage IJ, Borsboom GJ, Steyerberg EW, Essink-Bot ML (2009) Prostate cancer-specific anxiety in Dutch patients on active surveillance: validation of the memorial anxiety scale for prostate cancer. Qual Life Res 18(8):1061-1066

48. Kenfield SA, Chang ST, Chan JM (2007) Diet and lifestyle interventions in active surveillance patients with favorable-risk prostate cancer. Curr Treat Options Oncol 8(3):173-196

49. Brookman-May SD, Campi R, Henríquez JD, Klatte T, Langenhuijsen JF, Brausi M, ..., Minervini A (2019) Latest evidence on 
the impact of smoking, sports, and sexual activity as modifiable lifestyle risk factors for prostate cancer incidence, recurrence, and progression: a systematic review of the literature by the European Association of Urology Section of Oncological Urology (ESOU). Eur Urol Focus 5(5):756-787
50. Campbell MK, Carr C, DeVellis B, Switzer B, Biddle A, Amamoo MA, ..., Sandler R (2009) A randomized trial of tailoring and motivational interviewing to promote fruit and vegetable consumption for cancer prevention and control. Ann Behav Med 38(2):71-85

Publisher's note Springer Nature remains neutral with regard to jurisdictional claims in published maps and institutional affiliations. 